Cahiers de la recherche sur les droits fondamentaux

$4 \mid 2005$

Quel avenir pour la laïcité cent ans après la loi de 1905 ?

\title{
L'organisation des rapports entre État et religion en Turquie
}

\section{Élise Massicard}

\section{(2)enEdition}

\section{Journals}

Édition électronique

URL : https://journals.openedition.org/crdf/7333

DOI : $10.4000 /$ crdf.7333

ISSN : 2264-1246

Éditeur

Presses universitaires de Caen

Édition imprimée

Date de publication : 1 décembre 2005

Pagination : 119-128

ISBN : 2-84133-250-0

ISSN : $1634-8842$

Référence électronique

Élise Massicard, "L'organisation des rapports entre État et religion en Turquie », Cahiers de la recherche sur les droits fondamentaux [En ligne], 4 | 2005, mis en ligne le 15 décembre 2020, consulté le 14 novembre 2022. URL : http://journals.openedition.org/crdf/7333 ; DOI : https://doi.org/10.4000/crdf 7333 


\title{
L'organisation des rapports entre État et religion en Turquie
}

\author{
Élise MASSICARD \\ (CNRS / CERAPS)
}

Introduction

I. Ambiguïté des relations entre État et religion dans la République de Turquie
A. Les réformes de la jeune république
B. Un contrôle étatique sur la religion
C. Un islam national comme appareil idéologique d'État

II. Débats, contestations, évolutions
A. Les années 1950 : multipartisme et politisation
B. Les années 1960 : libéralisation et reconnaissance du rôle de la religion
C. Les années 1980 : visibilisation, politisation, luttes sur la place de la religion

III. Conclusion

IV. Bibliographie

\section{Introduction}

La Turquie est souvent présentée comme le seul pays «musulman et laïc». Quel destin pour un pays héritier du plus grand empire musulman au monde, qui a réuni les centres musulmans les plus importants et exercé sa domination sur le monde islamique pendant plusieurs siècles! Istanbul reléguait alors Bagdad, Le Caire, Damas et Jérusalem mais aussi La Mecque au rang de villes provinciales. L'Empire ottoman n'a pas seulement été le siège plusieurs fois centenaire du califat; l'islam y était religion d'État et le socle de sa légitimation, aussi multiconfessionnel que fût l'Empire. Lui seul incarnait alors l'islam pour les Européens.

C'est dire à quel point cette «laïcité» turque interroge. Soulignons tout de suite que le parallèle souvent dressé avec la laïcité française est trompeur, même si l'inspiration existe bien, puisque le terme «laiklik» est créé en turc moderne dans les années 1920 à partir du vocable français. Cependant, il semble ici nécessaire de dépasser la fixation sur un thème émotionnellement chargé - en Turquie comme en France - pour mieux comprendre les rapports complexes et changeants entre État et religion en Turquie.

\section{Ambiguïtés des relations entre État et religion dans la République de Turquie}

\section{A. Les réformes de la jeune république}

C'est en rupture avec l'héritage théocratique ottoman que Mustafa Kemal a instauré en 1923 une république 
reposant sur une légitimité tout autre que religieuse. La jeune république est marquée par de nombreuses réformes prenant pour cible le religieux, et qui se distinguent par leur ampleur et leur rapidité.

Au niveau institutionnel tout d'abord, le gouvernement d'Ankara abolit le sultanat en 1922. Cette mesure équivalait à la fin de la légitimation religieuse de l'ordre politique, ainsi qu'à la distinction des attributions spirituelles du chef religieux de l'islam des pouvoirs temporels : le calife n'avait plus, dès lors, que des attributions spirituelles. Mais le 3 mars 1924, l'Assemblée nationale abolit en outre le califat. Cette décision suscite une levée de boucliers dans les milieux religieux turcs, et fait l'effet d'une bombe dans l'ensemble du monde musulman. Les autres réformes passées le même jour-comme la dissolution du ministère des Affaires religieuses et des fondations pieuses - passent presque inaperçues.

La proclamation de la République de Turquie, le 29 octobre 1923, consomme la rupture : il s'agit de la première expérience de régime républicain dans le monde musulman. Dans la nouvelle constitution, adoptée le 20 avril 1924, le groupe religieux de l'Assemblée parvient à introduire un article (article 2) stipulant que « la religion de l’État turc est l'islam ». En 1928, cet article est amendé, et la référence à l'islam supprimée ${ }^{1}$.

Les réformes de laïcisation concernent également le domaine juridique : les tribunaux religieux sont abolis en $1924^{2}$. En 1926, le gouvernement abolit la charia comme source de loi, ainsi que les lois qui lui sont liées, et les remplace par... une adaptation du code civil suisse. Nul besoin d'insister sur l'ampleur des changements ainsi induits dans la vie religieuse et familiale ou le statut des femmes. Polygamie et répudiation sont interdites et remplacées par le mariage et le divorce civils. Tous les adultes obtenaient ainsi le droit de changer de religion à leur gré; pratique, il est vrai, mal vue et peu répandue. Cette réforme marque une brèche importante dans l'islam dans la vie privée, même si son application est loin d'être égale ${ }^{3}$.

En outre, le 3 mars 1924, l'Assemblée nationale vote la loi $n^{\circ} 430$ sur l'unification de l'enseignement qui rattache toutes les institutions d'enseignement au ministère de l'Éducation nationale. Elle ferme ainsi les écoles coraniques (medrese $)^{4}$, ce qui met fin à la formation de nouveaux théologiens. Cependant, l'enseignement religieux reste officiellement inclus dans l'enseignement général; si les autorités s'en désintéressent de fait dans les lycées et les collèges, il est maintenu jusqu'en 1939 dans les écoles primaires de village.

Cette politique antireligieuse est souvent présentée comme une rupture ex nihilo d'avec l'Empire ottoman. Pourtant, ce dernier avait tôt fait coexister la loi islamique (eriat) et la législation impériale (kânun). En outre, les réformes engagées depuis le $\mathrm{xIx}^{\mathrm{e}}$ siècle par les élites ottomanes cherchant à comprendre les raisons de la suprématie occidentale et du déclin impérial, ont, au-delà de leur dimension technique, impliqué le renforcement de l'État et la marginalisation des autorités religieuses et de leurs compétences. On peut donc aussi replacer les mesures de la jeune république dans la continuité des réformes entamées au XIX ${ }^{\mathrm{e}}$ siècle, qui ont notamment restreint progressivement la compétence des tribunaux religieux. Fut ainsi promulguée en 1840 , en pleine période de « réorganisation» (Tanzimat, 1839-1876), un nouveau code pénal dont les dispositions, tout en s'inscrivant dans le cadre de la eriat, n'en furent pas moins influencées par le droit français. En outre, cet empire multiconfessionnel avait dû trouver des formes juridiques pour faire cohabiter différents groupes religieux. Les millet, organisation juridique et administrative des groupes confessionnels non musulmans monothéistes - héritiers de la dhimmitude musulmane $(z i m m \hat{\imath})$ - furent renforcés et institutionnalisés au cours du XIX ${ }^{\mathrm{e}}$ siècle. Ils bénéficiaient d'une large autonomie religieuse et légale, qui a ouvert la voie à la définition de compromis politiques et juridiques et à une distinction relative entre les sphères religieuse et mondaine. Les édits impériaux dits Hatt-i Hümayûn de 1839 et de 1856 promettaient un statut et un traitement égaux à tous les sujets ottomans sans distinction de croyance. En outre, le pouvoir de régler les affaires intracommunautaires dans chaque millet, jusqu'alors dans les mains des autorités religieuses respectives, fut progressivement transféré à des assemblées de laïcs. Les rapports entre État et religion furent également un objet important des débats d'idées et des réformes qui marquèrent la fin de l'Empire. À la veille de la Première Guerre mondiale, dans un contexte de centralisation et d'accroissement du contrôle étatique, le gouvernement Jeune-Turc issu de la révolution constitutionnelle de 1908 impulse une réduction des pouvoirs religieux dans les institutions étatiques : limitation en 1913 de l'aire d'intervention des tribunaux religieux; rattachement de tous les tribunaux au ministère de la Justice en 1915, contrôle des fondations pieuses (vakf) par le ministère des Finances, limitation des prérogatives ministérielles du chef des clercs, le cheikh-ül islam, en 1916. Exigée par les conditions de guerre, cette politique visait moins à limiter le champ d'intervention de l'islam qu'à mettre les institutions religieuses sous le contrôle de l'État ${ }^{5}$.

Outre la question de l'inscription de ces réformes dans une histoire plus large, se pose également la question de leur qualification. Elles sont souvent résumées sous le chapeau de «laïcisation». Certaines des « lois de réforme» des années 1920 et 1930 ont ainsi été inscrites a posteriori dans les constitutions de 1961 et de 1982 en tant que principes fondamentaux dont l'immuabilité vaut comme garantie " du caractère laïc de la République de Turquie » (Constitution de 1961, art. 153, Constitution de 1982, art.

\footnotetext{
1. Loi $\mathrm{n}^{\circ} 1222$ de révision de la constitution de 1924, 10 avril 1928.

2. Loi n ${ }^{\circ} 469$ du 8 avril 1924.

3. J. Marcou, J.-P. Burdy, «Histoire et actualité de la laïcité en France et en Turquie », Cahiers d'études sur la Méditerranée orientale et le monde turco-iranien, $\mathrm{n}^{\circ}$ 19, janvier-juin 1995, p. 17.

4. Les minorités religieuses reconnues par le traité de Lausanne (voir plus bas) pouvant conserver leurs établissements religieux propres.

5. J. Marcou, J.-P. Burdy, «Histoire et actualité... », p. 16.
} 
$174)^{6}$. Or, le terme turc même de laïcité (laiklik) est postérieur à ces réformes. Il apparaît à l'heure des premiers bilans tirés par le régime kémaliste lui-même ${ }^{7}$, pour mettre rétrospectivement en cohérence une série de mesures ayant des champs d'application distincts. Le terme n'apparaît dans le vocabulaire politique qu'en 1931 au sein de discussions lors du congrès du parti unique, le Parti républicain du Peuple, $\mathrm{CHP}^{8}$. Sont édictés lors de ce congrès, puis insérés dans le programme du parti, les six principes généraux du régime. Ce n'est qu'en 1937 - peu avant la mort d'Atatürk - que le terme de laïcité apparaît dans le vocabulaire institutionnel, par son insertion, avec les cinq autres grands principes du kémalisme définis en 1931, dans l'article 2 de la Constitution à l'occasion de la révision du texte initial ${ }^{9}$. Le nouvel article 2 stipule explicitement que «l'État turc est républicain, national, populiste, étatiste, laïc et réformiste ${ }^{10}$. Cependant, les textes juridiques employant ce terme en ont rarement donné une véritable définition.

Ce caractère laïc est d'abord interprété comme l'exclusion de la religion des affaires étatiques. Ainsi, le programme du parti de 1931 " prend pour principe que dans la gestion de l'État, tous les textes de lois, de règlements et d'ordonnances doivent être rédigés et appliqués en conformité avec les principes et les formes puisés aux sources scientifiques et techniques de la civilisation moderne et en fonction des besoins temporels. Puisque la conception religieuse est une affaire de conscience, le Parti considère l'exclusion des idées religieuses hors des affaires étatiques et temporelles et hors de la politique comme l'un des principaux facteurs du succès de notre Nation dans la voie du progrès ${ }^{11}$.

De même, lors de la révision constitutionnelle de 1937, le ministre de l'Intérieur S. Kaya précise que : « [...] avec la laïcité, nous voulons empêcher que la Religion influe sur les affaires du pays [...]. Les religions doivent rester dans les consciences et les mosquées, et ne pas se mêler des choses temporelles et de la vie du monde ${ }^{12}$.

Ces mesures visent à écarter la religion de la vie politique et publique, à la cantonner à la pratique personnelle. Au début des années 1930, le secrétaire général du parti, Recep Peker, éclaircit la place qui lui reste, dans la sphère privée : «[...] la laïcité ne signifie absolument pas l'absence de religion, ou la volonté de son absence. En Turquie, l'exercice du culte, ainsi que chacun le souhaite, est garanti par la Constitution. Un citoyen qui est religieux par conviction personnelle peut très bien, malgré sa fidélité à sa conscience, être sincèrement laïc ${ }^{13}$.

\section{B. Un contrôle étatique sur la religion}

Il faut cependant nuancer cette «laïcité», ou du moins ne pas l'assimiler directement à la laïcité française. En effet, les relations entre État turc et religion sont loin d'être aussi simples qu'il y paraît au premier abord. La Turquie ne connaît pas de véritable séparation entre État et religion, ou plutôt il s'agit d'une séparation à sens unique. Si la religion ne doit pas intervenir sur les affaires d'État, en revanche l'État doit intervenir sur les affaires religieuses. Selon les termes même du dictionnaire Larousse: «La laïcité en Turquie, reconnaissant d'un côté la séparation des affaires religieuses des affaires de l'État, mais exerçant à travers l'État un large contrôle et une large intervention sur les sujets intéressant la religion et ne permettant pas ni l'activité ni les regroupements religieux, porte en cela une particularité ${ }^{14}$.

Le jour même de l'abolition du califat, le 3 mars 1924, est en effet créée une Direction générale des fondations pieuses (Vakflar Genel Müdürlü̈̈) - ce qui équivaut à la mise sous tutelle de tous les biens anciennement gérés par les oulémas. Le même jour est surtout créée une Direction des affaires religieuses, le Diyanet leri Bakanl (ou DB), dont le directeur est nommé par le président de la République sur proposition du Premier ministre. Directement rattachée au cabinet du Premier ministre, cette institution est dotée de pouvoirs considérables : elle est chargée de réguler et d'administrer toutes les questions de croyance et de rituel musulmans, principalement dans le cadre de l'enseignement et de la pratique du culte. C'est le Diyanet qui nomme, destitue et procède à la fonctionnarisation des hommes de religion, les imams et muezzins, après avoir surveillé leur formation dans des écoles de prédicateurs. C'est également le Diyanet qui contrôle les mosquées, décide des prêches qui y sont lus chaque vendredi, et administre le pèlerinage à La Mecque. Le DB supervise enfin les ouvrages d'enseignement de l'islam, les livres scolaires. Le Diyanet symbolise toute l'ambiguïté d'un imposant appareil religieux créé pour protéger le principe de laiklik. Loin d'avoir coupé les ponts avec la religion, l'État continue donc de l'organiser et de l'enseigner. Producteur de normes religieuses qu'il essaie d'imposer à la société, l'État turc se veut le titulaire exclusif des affaires religieuses

6. G. Groc, «Textes officiels turcs sur la laïcité», Cahiers d'études sur la Méditerranée orientale et le monde turco-iranien, $\mathrm{n}^{\circ}$ 19, janvier-juin 1995 , $\mathrm{p}$. 315 .

7. Lors du fameux discours-fleuve (Nutuk) prononcé par Mustafa Kemal devant l'Assemblée nationale, en octobre 1927. Cf. G. Groc, «Textes officiels turcs...", p. 316.

8. Cumhuriyet Halk Partisi. Son premier nom est CHF, Cumhuriyet Halk Frkas, qui signifie la même chose. Créé en 1923, le parti change de nom lors de son quatrième congrès en 1935. Pour des raisons de simplicité toutefois, nous utilisons l'abréviation CHP pour l'ensemble de la période.

9. Loi ${ }^{\circ} 3115$ du 5 février 1937.

10. G. Groc, «Textes officiels turcs...», p. 320.

11. G. Jaeschke, « Der Islam in der neuen Türkei. Eine rechtsgeschichtliche Untersuchung», Die Welt des Islams, vol. 1, n 1-2, 1951, p. 149, cité et traduit par G. Groc, «Textes officiels turcs...», p. 319 .

12. G. Jaeschke, « Der Islam in der neuen Türkei... », p. 151.

13. Cité et traduit par G. Groc, «Textes officiels turcs... », p. 319.

14. Büyük Larousse ansiklopedisi, cité et traduit par G. Groc, «Textes officiels turcs... », p. 317. 
et l'autorité ultime en la matière. L'État entend exercer un contrôle total sur les nouvelles institutions religieuses qu'il substitue aux anciennes. Il ne s'agit pas d'un désinvestissement de l'État par rapport au religieux, mais d'un contrôle de la religion par l'État.

Ce paradoxe apparaît au grand jour lors d'un arrêt de la Cour constitutionnelle du 21 octobre 1971. En juillet 1970 est adoptée une loi sur la fonctionnarisation des employés de la fonction publique, touchant toutes les administrations, y compris le Diyanet. Un recours est alors soumis à la Cour constitutionnelle concernant la possibilité, pour un État laïc, d'inscrire au rang de ses fonctionnaires des employés ayant pour tâche l'enseignement de la religion musulmane. Dans son arrêt - l'un des seuls commentaires juridictionnels de la laïcité - la Cour constitutionnelle s'étend sur les rapports entre État et religion. Elle commence par rappeler que le principe de laïcité, réglementant les relations entre État et religion, dépend des spécificités propres à chaque religion, mais aussi à chaque État. Elle revient sur ce que doit la laïcité à l'héritage de l'institution ecclésiale en Europe, et sur ses différences avec l'islam où il n'existe pas de clergé, avant de traiter plus spécifiquement le cas turc:

En résumé, le principe de laïcité présent dans la Constitution de la République de Turquie signifie précisément:

a) l'adoption de principe que la religion ne gouverne ni n'influe sur les affaires de l'État;

b) la garantie constitutionnelle de la religion par la reconnaissance d'une liberté illimitée de religion en ce qui concerne la conviction religieuse, en rapport avec la vie spirituelle des individus;

c) l'adoption de limitations et l'interdiction d'une mauvaise utilisation et d'une exploitation de la religion, dans le but de protéger l'ordre public, la sécurité et les intérêts publics liés au domaine de la religion, contre ce qui sortirait du domaine de la vie spirituelle des individus et influencerait l'activité et le comportement de la vie sociale;

d) la reconnaissance d'un droit de regard de l'État, en tant que garant de l'ordre public et des droits publics sur les libertés et droits religieux ${ }^{15}$.

En vertu de quoi, elle argumente que c'est justement pour ne pas permettre la constitution d'une caste religieuse spécifique que l'État intègre des fonctionnaires religieux au régime commun aux employés des administrations.

Ironiquement, cette mainmise de l'État sur la religion n'est pas sans rappeler une tradition ottomane. L'Empire ottoman a en effet procédé à une institutionnalisation sans précédent de l'islam au sein de l'État. Au fur et à mesure de son opposition à la Perse safavide voisine, qui proclame le chiisme comme religion d'État en 1501, soutenue en outre par de nombreux sujets ottomans hétérodoxes, la Sublime Porte, pour des raisons politiques plus que par conviction religieuse, fait du sunnisme sa religion officielle et s'en fait le défenseur. Peu après, le sultan Selim I ${ }^{\mathrm{er}}$ (1512-1520) conquiert les villes saintes de l'islam, La Mecque et Médine, ce qui lui apporte le titre de protecteur des lieux saints. Il annexe également l'Égypte, siège du dernier calife abbasside qui est alors déporté à Istanbul. La question de savoir si les sultans ottomans prennent alors le titre de califes est contestée ${ }^{16}$, mais ils remplissent implicitement ce rôle et sont reconnus comme représentants suprêmes de l'oumma par de nombreux empires musulmans, jusqu'en Asie centrale et dans le Sud-Est asiatique.

C'est à cette période que les sultans institutionnalisent l'islam au sein de l'État. Soliman le Magnifique (15201566) crée ainsi la fonction de eyh-ül islam, sorte d'équivalent du patriarche grec orthodoxe, ayant autorité sur l'ensemble des clercs. Il met l'administration de l'Empire en accord avec la charia, et perfectionne le système de formation des ouléma, les medrese. La construction de cette espèce d'Église musulmane fortement hiérarchisée, liée à l'État et rétribuée par lui, n'a pas de précédent dans la tradition musulmane. Ainsi les souverains ottomans ont-ils par la suite signé de la formule «l'épée de l'islam et l'ombre de Dieu sur la terre », adaptant trait pour trait les institutions du patriarcat orthodoxe de Constantinople. Sur la longue durée, les réformes kémalistes apparaissent en fait comme une étape dans le processus de domestication de la religion par l'État.

Par rapport à l'Empire, la prétention de l'État à monopoliser le religieux se renforce même sous la République ${ }^{17}$. La première raison est que les non-musulmans, si présents durant l'Empire ottoman, sont très peu nombreux sous la République. Certes, le traité de Lausanne (1923) - qui s'inspire directement de la structure administrative ottomane du millet - leur confère un statut de minorité reconnue $^{18}$ et énonce, outre des droits équivalents à ceux dont jouissent les ressortissants des États, une série de droits positifs ${ }^{19}$. Mais ce statut concerne désormais une part minime de citoyens: la société a été homogénéisée sur le plan religieux par les massacres des Arméniens à la fin du XIX ${ }^{e}$ siècle et en 1915, l'échange des populations chrétiennes orthodoxes de Turquie contre les populations musulmanes de Grèce en 1922, l'émigration d'une partie des Juifs après la création de l'État d'Israël, et la fuite de la majorité des Grecs d'Istanbul en Grèce après

15. E. Hirsch, «Laizismus (laiklik) als verfassungsrechtlicher Begriff in der türkischen Republik», Orient, $\mathrm{n}^{\circ}$ 15, septembre $1974, \mathrm{p} .109$.

16. C'est au plus tard à la fin du Xviı e siècle que les sultans, à la recherche de légitimité, réaffirment vivement leur qualité de califes. Voir G. Veinstein, "Les origines du califat ottoman », Les Annales de l'autre islam, nº 2, 1994, p. 25-36.

17. J.-F. Bayart, «Les trajectoires de la République en Iran et en Turquie : un essai de lecture tocquevillienne », in Démocraties sans démocrates, G. Salamé (dir.), Paris, Fayard, 1994, p. 381.

18. L’inscription de la religion sur la carte d'identité est également issue de l'héritage du système des millet et des obligations du traité de Lausanne. Cf. J. Marcou, J.-P. Burdy, « Histoire et actualité... », p. 18.

19. Ces dispositions sont sans précédent en droit international : le traité de Lausanne a entériné l'acception contemporaine du terme "minorité ", même s'il constituait un cadre juridique $a d$ hoc, visant à régler dans l'urgence le contentieux gréco-turc après la déroute de l'armée grecque en Asie Mineure (1922); il ne visait aucunement à donner une définition et un contenu universels à la notion de minorité. 
les violences de septembre 1955. Sous la République, les Turcs se trouvent donc dans leur immense majorité soumis au Diyanet. La seconde raison est que l'État a tenté de restreindre toute expression divergente de l'islam, susceptible d'échapper à son contrôle. Ainsi, les confréries soufies sont interdites et les couvents fermés ou confisqués depuis $1925^{20}$. En revanche, les confréries ne sont pas remplacées par une institution centralisée chargée de les administrer et de les contrôler. Enfin, les courants religieux ont été violemment combattus aux débuts de la République et poussés vers la clandestinité.

\section{Un islam national comme appareil idéologique d'État}

Dans une large mesure, cette domestication de la religion implique son instrumentalisation par le régime kémaliste pour affermir la cohésion nationale, pour ainsi dire comme «appareil idéologique d'État ${ }^{21}$. Dans la perspective de création d'un État-nation, l'islam joue dans les faits le rôle de religion officielle et occupe une place de première importance dans la définition de l'identité nationale. Comme sous l'Empire, le Diyanet fonctionne paradoxalement comme une institution de légitimation du pouvoir et, dans ce cadre, doit mettre en pratique une version nationale de l'islam. Mais de quel islam s'agit-il ?

D’une manière générale, ces réformes ne visent pas à éradiquer l'islam, mais à le réformer pour en faire une religion éclairée et moderne. Elles s'inscrivent dans un projet plus vaste, qu'on peut qualifier de civilisationnel. Le kémalisme avait pour ambition de créer un nouvel homme turc dépouillé de tout signe marquant sa parenté avec la communauté musulmane - relégués à la sphère privée - mais se rapprochant au contraire de l'Europe, symbole de la civilisation. De nombreuses mesures, sans toucher directement la sphère religieuse, ont une dimension de rupture avec la religion d'origine arabe et l'histoire ottomane : le port du fez et du turban est interdit en 1925 et remplacé par un couvre-chef européen. Le voile n'est pas interdit, mais fortement critiqué. En 1928, l'alphabet arabe - considéré par les élites républicaines comme un signe d'arriération - est remplacé par les caractères latins. En 1932, c'est la réforme linguistique, chasse aux termes d'origines arabe et persane; de même, l'appel à la prière, jusqu'alors en arabe, devra se faire en turc. En 1934 sont adoptés les patronymes européens et les titres à dimension religieuse, tels ceux de efendi, hac, hoca ou mollah abolis et remplacés par monsieur (bay) et madame (bayan). Même les rythmes de vie sont réformés, avec le remplacement du calendrier musulman par le calendrier grégorien (1925), la réforme des mesures de temps (1928), puis l'adoption du dimanche comme jour de repos hebdomadaire (1935). Ces mesures entraînent une vive opposition dans le pays. Dans ce cadre, l'islam ne devait pas être éradiqué, mais modernisé et purifié de ses éléments « rétrogrades» ou « obscurantistes ». Estimant que les mausolées de saints (türbe) sont des lieux de pratiques magiques et superstitieuses, donc ennemis du progrès et de la civilisation, les kémalistes les ferment en $1925^{22}$; le culte des saints, les devins et les guérisseurs sont interdits. On voit ici que le contrôle étatique sur la religion touche également à la sphère privée.

Une autre dimension de cet islam officiel est le monisme. Si l'islam acquiert un rôle de ciment de la nation, c'est dans le cadre d'un projet de construction d'une communauté nationale qui a pour particularité son caractère unitaire, aveugle à tout élément particulariste. De ce fait, cet islam officiel ignore la pluralité confessionnelle, pourtant bien réelle, de la Turquie. En pratique, si le kémalisme a souvent eu recours aux références religieuses comme source de légitimation, c'est toujours au sunnisme hanéfite qu'il a fait appel, l'érigeant ainsi en « confession officielle par défaut ${ }^{23}$. «La République a fait de l'islam sunnite une sorte d'appareil idéologique d'État, à l'exclusion de tendances plus hétérodoxes: les alévis, les chaféites kurdes, certaines confréries plus mystiques mais moins ritualistes [...], à l'exclusion aussi des mouvements islamistes » ${ }^{24}$.

La « laïcité» turque n'implique donc pas de pluralisme ni de libéralisme religieux, ne signifie pas la reconnaissance de l'égalité ni même de la pluralité confessionnelle; mais au contraire l'officialisation d'une seule confession, sunnite hanéfite. Là aussi, on est obligé de constater une certaine continuité avec l'Empire, qui reconnaissait certes la diversité religieuse, mais uniquement pour les non-musulmans: reçurent le statut de millet les communautés religieuses reconnues comme "gens du Livre» par l'islam, juifs et chrétiens - avec, pour la chrétienté, ses différentes confessions et Églises, parfois nationales ${ }^{25}$. Mais les musulmans hétérodoxes - notamment les descendants de ceux qui soutenaient la Perse safavide au Xvi ${ }^{\mathrm{e}}$ siècle, les alévis sortaient de ce cadre: illégitime dans le cadre même de l'islam, leur différence n'était donc ni reconnue ni institutionnalisée, ce que perpétue la République.

$\mathrm{Au}$-delà de l'aspect spectaculaire des réformes et de l'affirmation du principe de «laïcité», force est donc de constater les continuités qui unissent Empire ottoman et République dans la gestion du religieux: les institutions ont la mainmise sur le religieux, au point de pouvoir conclure à l'institutionnalisation d'une religion officielle.

20. On estime que 776 tekke (couvents de derviches) ont été touchés par cette réforme.

21. J. Marcou, J.-P. Burdy, « Histoire et actualité... », p. 18.

22. On estime que 905 türbe ont été fermés.

23. H. Bozarslan, «Au-delà de l'abolition du Khalifat. Laïcité, État-Nation et contestation kurde », Les Annales de l'autre islam, n 2, 1994, p. $225-235$.

24. O. Abel, «La condition laïque. Réflexions sur la laïcité en Turquie et en France», Cahiers d'études sur la Méditerranée orientale et le monde turco-iranien, $\mathrm{n}^{\circ} 19$, janvier-juin 1995, p. 39.

25. La référence à ce sujet est Christians and Jews in the Ottoman Empire: The functioning of a plural society, B. Braude, B. Lewis (éd.), New York, Holmes \& Meier, 1982, 2 vol. 


\section{Débats, contestations, évolutions}

Il est important de souligner que, dès l'origine, ces réformes ont été contestées : la place de la religion dominante dans l'État et la société, l'existence et le financement de l'enseignement religieux, la laïcité du système scolaire public, le port du türban ont été objets de débats virulents. Les relations entre État et religion n'ont pas cessé, depuis les débuts de la République, d'être âprement discutées et contestées. De ce fait, elles ont connu des évolutions et des inflexions importantes selon les aléas politiques.

Au début de la République, le manque de base sociale large aux réformes a provoqué de nombreuses résistances. C'est probablement aussi les soulèvements au nom de la religion ou du rétablissement du califat qui ont provoqué la répression de tout groupement religieux susceptible de constituer un cadre de mobilisation en dehors des institutions; par exemple, la révolte dirigée par le nakibendi cheikh Saïd en 1925 a eu pour suite l'interdiction des confréries. L'imposition du principe de laïcité l'a emporté sur le pluralisme et la démocratie, car les élites modernistes considéraient que la souveraineté populaire risquait de provoquer le retour à l'islam ${ }^{26}$.

\section{A. Les années 1950 : multipartisme et politisation}

La Deuxième Guerre mondiale amène une inflexion massive. Les débats sur la nécessité de l'enseignement religieux pour la moralisation de la jeunesse se nourrissent de la peur grandissante du communisme, lui-même aux portes du pays. Mais cet enseignement pose le problème des formateurs, dont la pénurie est flagrante. Pour ne pas s'en remettre à des enseignants douteux et non contrôlables, les institutions doivent mettre en place un enseignement religieux conforme aux normes républicaines, donc un cursus d'enseignement professionnel qui passe par la multiplication des lycées d'imams-prédicateurs (imamhatip) dès 1948. Ce paradoxe condamne l'État, pour tenter de contrôler la religion et d'en imposer sa vision «laïque », à multiplier les instances d'éducation religieuse ${ }^{27}$. En 1950, vingt mausolées de personnages illustres sont réouverts, avec possibilité conditionnelle de pèlerinage.

Parallèlement, pour ne pas perdre le contrôle de cette brèche ouverte, les peines encourues pour propagande religieuse sont aggravées. Ainsi, l'article 163 du Code pénal, adopté en mars 1926, est révisé en juin 1949; il inclut désormais le terme de laïcité :

Paragraphe 1: «Quiconque fonde, organise, coordonne ou dirige une association dans le but, opposé à la laïcité, de rendre l'organisation fondamentale de l'État - sociale, économique, politique ou juridique - conforme à des principes ou des croyances religieuses, même de manière temporaire, sera puni d'une peine de prison ».
Paragraphe 3: «Quiconque s'adonne à une activité de propagande ou use d'influence de quelque manière que ce soit dans le but, opposé à la laïcité, de rendre l'organisation fondamentale de l'État - sociale, économique, politique ou juridique conforme à des principes ou des croyances religieuses, même de manière temporaire, ou à des fins politiques ou encore avec le but de créer ou de conforter un intérêt politique par utilisation de la religion ou de sentiments religieux ou d'objets tenus pour sacrés par la religion, sera puni d'une peine de prison».

Si le besoin de sanctionner plus durement la propagande religieuse se fait sentir, c'est aussi en raison de l'introduction du multipartisme en 1946. Est ainsi instaurée une logique politique nouvelle: il s'agit désormais pour chaque parti de convaincre les électeurs; dès lors, il est nécessaire de prendre en compte les attentes de la population. Dans ce contexte, la religion devient un argument électoral de poids, une concession aux demandes de la population utilisée par tous les partis, notamment la droite conservatrice, qui se pose comme alternative au CHP. Tout en acceptant le principe de séparation des affaires religieuses et des affaires d'État, ils précisent que la laïcité ne doit en aucun cas équivaloir à une hostilité vis-à-vis de la religion, réaffirment la liberté de religion, de croyance et de culte, distinguent entre « réaction religieuse » et « foi » ou «piété ». Les valeurs islamiques s'expriment désormais plus facilement par ces partis alternatifs, qui parviennent au pouvoir, notamment durant toute la décennie 1950.

De manière significative, les premières mesures du Parti démocrate (DP) concernent l'assouplissement des « lois de réforme » : c'est ainsi que l'appel à la prière est de nouveau lu en arabe dès mai 1950 ; que les normes vestimentaires sont assouplies ou au moins qu'une plus grande tolérance se fait jour. Les cours d'éducation religieuse sont introduits à l'école publique dans le secondaire en 1956, même s'ils restent facultatifs. Est également envisagée leur introduction au lycée, mais cette dernière est retardée jusqu'en 1967, faute de personnel compétent. Le contenu des cours reste fidèle à l'esprit kémaliste de développer l'utilité de la religion dans la vie personnelle, sociale et nationale. Ils visent à renforcer l'unité nationale, et à montrer que l'islam est une religion rationnelle qui encourage la civilisation et le progrès. L'une des exigences formulées par l'armée au Premier ministre quelques jours avant le coup d'État qui met fin au règne du DP était justement de renoncer à exploiter politiquement la religion.

\section{B. Les années 1960 : libéralisation et reconnaissance du rôle de la religion}

La période issue du coup d'État militaire du 27 mai 1960 marque cependant un tournant : la volonté des officiers d'initier des débats autour de sujets jusque-là considérés comme dangereux établit un climat nouveau, favorisé

26. N. Göle, "Laïcité, modernisme et islamisme en Turquie», Cahiers d'études sur la Méditerranée orientale et le monde turco-iranien, ${ }^{\circ}$ 19, janvier-juin 1995 .

27. Les cours facultatifs de religion sont rétablis à l'école primaire le 15 février 1949. Une faculté de théologie est créée à Ankara le 4 juin 1949. 
par la Constitution relativement libérale de 1961, qui garantit les libertés d'expression, d'association, de conscience et de foi. Son article 19 stipule ainsi que toutes les cérémonies religieuses qui ne vont pas à l'encontre de l'ordre public ou de la morale générale sont libres.

Cependant, le caractère laïc de l'État est réaffirmé par son article 2 et érigé - parmi d'autres - par l'article 8 au rang de principe supérieur aux normes juridiques. Cet article est complété par l'introduction d'une référence aux « lois de la réforme ${ }^{28}$ dans le préambule, ainsi que dans l'article 153. L'article 57 sur les partis politiques fait référence au principe laïc, et renvoie en outre à un dispositif législatif (loi sur les partis politiques no $648 \mathrm{du} 13$ juillet 1965) qui explicite (articles 83 à 107) la protection du fondement de l'État laïc et des réformes d'Atatürk. C'est d'ailleurs en protection de cette loi qu'un premier arrêt est rendu le 20 mai 1971 par la Cour constitutionnelle (Anayasa Mahkemesi), ayant pour objet le jugement de « comportements contraires au principe de laïcité». Cet arrêt prononce l'interdiction du Parti de l'Ordre national $^{29}$.

L'article 154 de la Constitution de 1961 intègre le DIB, jusque-là rattaché au Premier ministre, à l'administration générale, et le soumet aux règles de droit public. Ses fonctions, relevant du domaine de la loi, sont définies et élargies par la loi $\mathrm{n}^{\circ} 633$ adoptée le 22 juin 1965: «Administrer les croyances, les pratiques et les principes moraux de la religion musulmane; éclairer la société à propos de la religion et gérer les lieux de culte». Il ne s'agit donc plus seulement, comme dans les premières années de la République, de la gestion des lieux de culte et de la formation, nomination et rétribution des imams et autres personnels religieux. À cela s'ajoute désormais le devoir d'informer et "d'éclairer» la société sur la religion. Le DIB publie ainsi des ouvrages et une revue, et diffuse toute une littérature de vulgarisation et de sensibilisation sur des thèmes religieux. Il donne son avis pour l'importation d'ouvrages religieux et assure la traduction du Coran. Il développe également des centres d'apprentissage de la lecture coranique. Mais il n'a aucune attribution théologique et ne contrôle pas les écoles ni la faculté de théologie, conformément au principe d'unité de l'enseignement, et au grand dam des islamistes.

À ces nouvelles fonctions correspond également un essor de l'institution elle-même : simple bureau de quelques milliers de personnes à sa création, cette Direction n’a cessé de s'agrandir. En 1981, son budget dépassait celui du ministère de l'Industrie, et n'a cessé d'augmenter depuis lors pour atteindre en 2002 près de 400 millions d'euros. D'après ses propres chiffres, le DIB gère actuellement plus de 75000 mosquées, près de 90000 fonctionnaires et 75000 employés et gère 3664 centres où presque 120000 enfants suivent des cours de lecture coranique ${ }^{30}$.

\section{Les années 1980 : visibilisation, politisation, luttes sur la place de la religion}

Cette évolution est renforcée après le coup d’État de 1980 : l'armée turque, tout en réprimant certains mouvements d'inspiration religieuse, a de nouveau favorisé la référence religieuse comme ciment national. Dans la nouvelle constitution promulguée en 1982 sous le régime militaire, le principe de laïcité occupe sa place habituelle: le « caractère laïc » de l'État turc est énoncé par l'article 2 de la Constitution de 1982; beaucoup d'articles (sur les partis politiques, la radio...) s'y réferent ensuite, ou plus généralement aux « caractères fondamentaux» de la République définis dans les trois premiers articles du texte constitutionnel, et déclarés inaltérables par l'article 4. L'article 10 de la Constitution prévoit que chacun est égal devant la loi, sans considération de religion, de confession, ou du même ordre, et que l'État doit rester neutre face aux religions et refuser l'adoption d'une religion officielle. Chacun possède la liberté de conscience, de croyance et de conviction religieuses. Les prières, les cérémonies et rites religieux sont libres à condition de ne pas être contraires aux dispositions de l'article 14 . Nul ne peut être astreint à prendre part à des prières, des cérémonies ou rites religieux, ni à divulguer ses croyances et convictions religieuses, et nul ne peut être blâmé ni incriminé en raison de ses croyances ou convictions. Nul ne peut, de quelque manière que ce soit, exploiter la religion, les sentiments religieux et les choses considérées comme sacrées par la religion.

Ce texte fait cependant plus que reprendre les dispositions de plusieurs textes antérieurs; il introduit également une obligation généralisée de l'éducation religieuse (art. 24). L'enseignement de la culture religieuse et de la morale figure parmi les cours obligatoires dispensés dans les établissements scolaires du primaire et du secondaire ; il est dispensé sous la surveillance et le contrôle de l'État. Les familles sont obligées de déclarer leur religion et leurs enfants musulmans de suivre ces cours. Ce texte traduit la volonté d'une recomposition autour d'un consensus national anticipé à partir de principes moraux reconnus dans l'islam. La laïcité reste une référence fondamentale, mais la doctrine étatique intègre de plus en plus l'islam national.

En outre, dans les années 1980, sous l' «ère Özal » ${ }^{31}$, "président croyant» à la pratique religieuse visible, les mesures législatives et administratives favorables à l'islam

28. À savoir, la loi de l'unification de l'enseignement, celle du chapeau, la loi sur la fermeture des couvents et mausolées, le mariage civil, la réforme de l'écriture, l'abolition des titres ottomans et la réforme vestimentaire.

29. En 1970, Necmettin Erbakan fonde le Milli Nizâm Partisi (Parti de l'Ordre national), largement considéré comme le premier parti islamiste de Turquie. Il s'agit plus d'un parti de la morale et de bienséance, des valeurs nationales et spirituelles, du respect des traditions et de l'ordre, que d'un parti véritablement islamiste visant à l'instauration de la charia, même si certains de ses membres sont plus radicaux.

30. À l'étranger, depuis le milieu des années 1980, le Diyanet dispose de représentants dans les ambassades et les consulats. Avec le développement d'une migration de Turquie dans les pays européens et avec la prise de conscience que sa présence est amenée à perdurer, il a été décidé d'envoyer des représentants du Diyanet dans les grandes villes de migration turque en Europe, qui est également un instrument de politique étrangère.

31. Turgut Özal fut Premier ministre de 1983 à 1989, puis président de la République jusqu’à sa mort en 1993. 
se sont succédées. Une tolérance accrue se manifeste à l'égard des confréries ou des associations religieuses, et le port du foulard islamique (türban) se fait de plus en plus visible dans l'espace public. Alors que la loi datée du 3 décembre 1934 interdit le port d'un habit religieux, quelle que soit la religion, en dehors des lieux de culte et des cérémonies religieuses, l'essor du port du türban fait l'objet de vifs débats. Pour les uns, il est une obligation et / ou une manifestation liée à l'identité religieuse, pour les autres, il est un symbole de l'islam politique, menaçant la laïcité, la paix civile et les droits des femmes. La multiplication des signes religieux et la tolérance accrue donnent lieu à de nombreux incidents, oppositions et résistances. Ainsi, le Conseil d'État précise-t-il le 13 décembre 1984 que : «audelà d'une simple habitude innocente, le foulard est en train de devenir le symbole d'une vision contraire aux libertés de la femme, à l'égalité des sexes et aux principes fondamentaux de la république ». La question est encore plus sensible concernant le port du foulard dans les administrations et les établissements d'enseignement public. Ainsi, le règlement du 22 juillet 1981, adopté par le Conseil des ministres, impose aux personnels travaillant dans les organismes et institutions publics ainsi qu'aux agents et étudiants rattachés aux ministères une tenue vestimentaire simple, sans excès et «contemporaine» (çada). Les femmes (y compris les étudiantes) doivent être non voilées dans les établissements d'enseignement. Ce règlement est complété par une circulaire datée du 20 décembre 1982, adoptée par le Conseil de l'enseignement supérieur, qui interdit le port du foulard islamique dans les salles de cours. Un règlement de 1985 prévoit des sanctions disciplinaires. Dans l'arrêt du 7 mars 1989, la Cour constitutionnelle annule une loi du gouvernement Özal mettant fin à l'interdiction de la tenue islamique sur les campus universitaires. Elle précise que, en dehors du cadre intime réservé à l'individu, la liberté de manifester la religion pouvait être restreinte pour des raisons d'ordre public dans le but de préserver le principe de laïcité. Le 9 avril 1991, la Cour constitutionnelle déclare : «dans les établissements de l'enseignement supérieur, se couvrir le cou et les cheveux avec un voile ou un foulard pour des raisons de conviction religieuse est contraire aux principes de laïcité et d'égalité».

À la diversification des pratiques et à la tolérance accrue à l'égard de la religion répond ainsi une crispation du droit et de certaines institutions, aboutissant parfois à des luttes entre institutions sur la place de la religion. L'article $163 \mathrm{du}$ Code pénal, symbole juridique de la laïcité militante, qui permet de «requérir de lourdes peines de prison contre tous ceux qui, exploitant à des fins politiques la religion, les sentiments religieux ou les choses sacrées, incitent les masses à accomplir des actes qui mettent en danger la sécurité de l'État ou créent des associations dans ce but, même s'ils ne dépassent pas le stade de la tenta- tive ", est aboli par Özal en 1991; mais, même après son abolition, la plupart de ses dispositions perdurent, reformulées en tout ou en partie dans d'autres textes.

La virulence accrue des luttes entre institutions au sujet de la place de la religion n'est certainement pas étrangère au poids croissant sur la scène politique des partis se réclamant de l'islam. Le RP (Refah Partisi, Parti de la prospérité) connaît des succès électoraux importants aux municipales de 1994 puis aux législatives de 1995, où il devient la première force politique du pays; il accède au gouvernement, dans le cadre d'une coalition, en 1996. Mais les oppositions et résistances sont fortes; le 28 février, le Conseil national de sécurité dominé par les militaires pose un ultimatum au gouvernement, l'invitant à éradiquer toute manifestation islamiste dans la société, puis le pousse à la démission en mai. Le 9 janvier 1998, le RP est dissous par un arrêt de la Cour constitutionnelle (anayasa mahkemesi), pour utilisation de symboles religieux à des fins politiques - son successeur, le FP (Fazilet Partisi, Parti de la Vertu), connaîtra le même sort le 22 juin 2001. La Cour estimait que le projet politique du RP était dangereux pour les droits et libertés garantis par la Constitution turque, dont la laïcité, et qu'il avait des chances réelles de mettre en application son programme s'il accédait au pouvoir. Le parti porte alors l'affaire devant la CEDH. Dans l'arrêt du 13 février 2003, Refah Partisi et autres c / Turquie, celleci a estimé que la dissolution du RP ne violait pas la Convention européenne, compte tenu de la consécration de la laïcité dans la Constitution turque et du danger réel que constituait ce parti pour les droits et libertés en Turquie s'il parvenait au pouvoir. Elle a estimé que le principe de laïcité « était assurément l'un des principes fondateurs de l'État, qui cadre avec la prééminence du droit et le respect des droits de l'homme et de la démocratie. Une attitude ne respectant pas ce principe ne sera pas nécessairement acceptée comme faisant partie de la liberté de manifester sa religion et ne bénéficiera pas de la protection qu'assure l'article 9 de la Convention ».

L'intervention croissante de la CEDH dans ces contentieux ayant pour enjeu les relations entre État et religion ne se limite pas à cette affaire, mais constitue une tendance générale depuis les années $1990^{32}$. Dans cette affaire comme dans les autres, la Cour se range du côté de la défense de la laïcité officielle. Ainsi, dans l'arrêt Kalaç contre Turquie du $1^{\text {er }}$ juillet 1997, elle a validé la sanction disciplinaire prononcée contre un militaire se livrant au prosélytisme religieux. Il en est de même concernant la question du port du foulard: dans l'arrêt Karadum contre Turquie du 3 mai 1993, la CEDH, après avoir relevé l'existence d'un enseignement privé parallèle à l'enseignement public, admet l'interdiction du port de signes religieux dans les établissements publics d'enseignement supérieur turcs si celui-ci nuit à l'objectif de protection des droits et des libertés d'autrui, de l'ordre et de la sécurité publique.

32. En tant que membre du Conseil de l'Europe, la Turquie signe la Convention européenne des droits de l'homme le 4 novembre 1950 et la ratifie le 18 mai 1954. Elle ne ratifie cependant pas tous les protocoles suivants. De même, elle n'accepte le droit de recours individuel (article 25) que le 28 janvier 1987 et ne reconnaît la juridiction obligatoire de la CEDH (article 46) que le 22 janvier 1990. En partie en raison de ces évolutions, les requêtes déposées contre l'État turc augmentent en flèche dans les années 1990 : inférieures à la centaine annuelle jusqu'en 1991, elles dépassent les 200 en 1995 , puis les 500 en 1996. 
En juillet 1998, l'étudiante en médecine Leyla Ahin, qui souhaitait porter le voile à l'Université d'Istanbul, a saisi la CEDH au motif que l'interdiction du port du foulard islamique dans les établissements d'enseignement supérieur constituerait une violation des droits et libertés (articles $8,9,10$ et 14 de la Convention). Le jugement, rendu à Strasbourg le 29 juin 2004, lui donne tort. La Cour juge respectueuse des valeurs sous-jacentes à la Convention, la conception exprimée par l'arrêt de la Cour constitutionnelle turque du 7 mars 1989, selon laquelle la laïcité constitue le garant des valeurs démocratiques et des principes d'inviolabilité de la liberté de religion pour autant qu'elle relève du for intérieur, et de l'égalité des citoyens devant la loi. Sur cette base, la CEDH constate ensuite que la sauvegarde du principe de laïcité peut être considérée comme nécessaire à la protection du système démocratique de la Turquie. Elle estime en outre que l'on ne saurait faire abstraction de l'impact que peut avoir le port du foulard (perçu comme une obligation religieuse contraignante) sur ceux et celles qui ne le portent pas. Une limitation en la matière peut donc répondre à un «besoin social impérieux » tendant à atteindre les deux buts légitimes que sont la laïcité et les droits des femmes, d'autant plus que ce symbole religieux a acquis, au cours de ces dernières années en Turquie, une portée politique. La Cour conclut que la réglementation de l'Université d'Istanbul, qui soumet le port du foulard islamique à des restrictions, est justifiée dans ses principes et proportionnée aux buts poursuivis et que, partant, il n'y a pas eu violation de l'article 9 de la Convention. L'approche de la Cour repose sur une reconnaissance des traditions de chaque pays, sans chercher à imposer un modèle uniforme de relations entre l'Église et l'État.

\section{Conclusion}

Les relations entre État et religion en Turquie sont marquées par de nombreux paradoxes, incarnés par le Diyanet : censée veiller à l'apolitisme de l'islam, cette institution est aussi critiquée comme vecteur de pénétration des islamistes dans l'appareil d'État. Certains militants de l'islam politique voient en lui une structure disponible qui attend d'être conquise, et certains défenseurs de la laïcité y voient un garde-fou salutaire contre un islam moins contrôlé. De l'autre côté, certains intellectuels laïques et certains militants islamistes se rejoignent pour réclamer sa suppression, pour séparer État et religion de manière plus stricte, ou au contraire pour secouer la tutelle pesante que les institutions exercent sur l'islam et agrandir la marge d'autonomie de celle-ci.
Cette question est, depuis des décennies, au centre des débats politiques et sociaux en Turquie; elle est l'objet de luttes entre courants et partis politiques, mais aussi entre institutions : armée et justice restent les principaux défenseurs d'une conception restrictive, peu tolérante de la laïcité. Soumises aux aléas politiques, les relations entre État et religion sont susceptibles de continuer à changer. D'une part, l'AKP (Adalet ve Kalknma Partisi, Parti de la Justice et du Développement), le parti actuel de gouvernement, d'inspiration religieuse mais avant tout libérale et conservatrice, a permis de désamorcer certaines tensions en tentant d'éviter les oppositions frontales avec les institutions; d'autre part, la place de la religion attire l'attention - et souvent les critiques - des observateurs de la Turquie à l'étranger, dont l'importance ne saurait être sous-estimée dans ce contexte de débats virulents sur l'intégration de la Turquie à l'Union européenne.

\section{Bibliographie}

Berkes N., The Development of Secularism in Turkey, Montréal, McGill University Press, 1964.

DÂver B., Türkiye Cumhuriyetinde Laiklik, Ankara, Siyasal Bilgiler Fakültesi, 1955.

DinçKol B., 1982 Anayasas çerçevesinde ve Anayasa Mahkemesi Kararlarnda Lâiklik, Istanbul, Kazanc hukuk yay., 1992.

ЈАсов X., L'Enseignement religieux dans la Turquie moderne, Berlin, Klaus Schwarz, 1982.

Massicard É., "L'islam en Turquie, pays "musulman et laïc” ", in La Turquie aujourd'hui, un pays européen?, O. Roy (dir.), Paris, Universalis, 2004, p. 55-67.

Tarhanl Tar B., Müslüman toplum, «laik» devlet, Türkiye'de Diyanet leri bakanl, Istanbul, Afa, 1993.

Uur A., «L'ordalie de la démocratie en Turquie. Le projet communautarien islamiste d'Ali Bulaç et la laïcité », Cahiers d'études sur la Méditerranée orientale et le monde turco-iranien, ${ }^{\circ}$ 19, janvier-juin 1995.

Zarcone T., La Turquie moderne et l'islam, Paris, Flammarion, 2004. 